\title{
Uso de drogas inyectables y VIH en Rosario (Argentina) Fundamentos para la reducción de daños*
}

\author{
INCHAURRAGA, S. **; SIRI P.*** \\ * Psicóloga. Master en Drogodependencias por la Universidad de Barcelona. Directora del Centro de Estudios Avanzados en Drogadependencias y SIDA \\ de la Universidad Nacional de Rosario y del Servicio Asistencial para Drogadependientes del Centro Regional de Salud Mental de Rosario. \\ Presidente de la Asociación de Reducción de Daños de la Argentina (ARDA). \\ ***Psiquiatra. Doctor en medicina. Coordinador del Observatorio Epidemiológico en Adicciones del CEADS - UNR. \\ Investigador Asociado del CEADS-UNR (CRSM) \\ Enviar correspondencia a: \\ Silvia Inchaurraga sinchaur@agatha.unr.edu.ar
}

\section{Resumen:}

Introducción: El uso de drogas inyectables (UDI) es una práctica ampliamente difundida en la ciudad de Rosario, en Argentina. Desde hace más de una década, el compartir equipos de inyección fue el factor responsable de contagio en más de la mitad de los casos de VIH registrados en Rosario.

Objetivos: Realizar una aproximación a la evaluación de la situación del uso de drogas inyectables y sus consecuencias en el marco metodológico del RAR (método de Evaluación y Respuesta Rápida) recomendado por la OMS, para orientar las intervenciones de las políticas de salud en drogadependencias en la ciudad.

Material y Métodos: El estudio abarca estudios epidemiológicos previos o relacionados, encuestas por método directo (consultantes) e indirecto (muestreo en red), entrevistas directas a consultantes y triangulación de la información.

Resultados: EI UDI es un fenómeno frecuente en nuestro medio, se ha estimado que más de 3.000 personas podrían haber contraído el VIH por esta vía en Rosario desde el inicio de la epidemia. La cocaína es utilizada por el 100\%, una parte minoritaria utiliza opiáceos. A través de un muestreo en red se recolectaron datos sobre 170 usuarios de drogas inyectables, el $63 \%$ de los cuales eran $\mathrm{VIH}(+)$, el 70 \% comparte equipos de inyección. El $89 \%$ de este grupo no concurría a ningún tratamiento para su adicción, el 13\% tiene menos de 20 años. Sólo 20\% de los UDINIH (+) realizan control y tratamiento de su infección.

Conclusiones: Si bien estudios más profundos e intervenciones urgentes sobre este grupo deberán ser puestas en marcha a la brevedad, tanto los datos oficiales como la investigación exploratoria realizada, nos permiten fundamentar la importancia de las estrategias de reducción de daños. Un modelo que aunque resistido por las políticas oficiales de drogas, venimos poniendo en marcha en nuestra ciudad desde nuestro Centro de Estudios Avanzados en Drogadependencias y SIDA de la Universidad Nacional de Rosario y nuestro Servicio Asistencial para Drogadependientes del Centro Regional de Salud Mental "Agudo Avila".

Palabras clave: drogas inyectables, tratamiento, sida, reducción del daño, epidemiología, investigación cualitativa, Argentina.

\section{Summary}

Introduction: Injecting drug use (IDU) is widespread in the city of Rosario in Argentina. For more than a decade, the shared use of injection equipment has been the factor responsible for contagion in more than half the cases of HIV registered in Rosario.

Objectives: To carry out an approach to the evaluation of the situation in injecting drug use and its consequences within the methodological framework of RAR (an evaluation and rapid response method) recommended by the WHO in order to focus health policies on interventions in drug dependency in the city.

Material and methods: The study includes prior or related epidemiological studies, surveys using direct methods (consultants) and indirect (network sample), direct interviews with consultants and triangulation of the information.

Results: The IDU is a frequent phenomenon in our environment and it has been estimated that more than 3,000 people could have contracted HIV by this means in Rosario since the commencement of the epidemic. $100 \%$ use cocaine, a minority use opiates. Using network sampling, data was collected on 170 intravenous drug users, $63 \%$ of whom were $\mathrm{HIV}(+), 70 \%$ shared injection equipment, $89 \%$ of this group were not undergoing any treatment for their addiction and $13 \%$ were under 20 years of age. Only $20 \%$ of the IDU/HIV(+) undertook any monitoring and treatment of their infection.

Conclusions: Although there must be more in-depth studies and urgent interventions made in this group as soon as possible, both the official data and the exploratory research enable us to emphasise the importance of harm reduction strategies. Although there is resistance to these in the official drug policies, we are beginning to implement them from our Advanced Studies in drug dependency and AIDS Centre of the Rosario National University, and our drug dependents Assistance Service in the Agudo Avila Mental Health Regional Centre.

Key words: injecting drugs, treatment, aids, harm reduction, epidemiology, qualitative research, Argentina

\footnotetext{
* La versión completa original del estudio al que hace referencia el presente artículo fue presentada en la X International Conference on Drug Related Harm, Ginebra, Suiza, 21 - 25 de Marzo de 1999, bajo el título "Epidemiologic Profile of Drug Addiction on IDU population in Rosario City, Argentina. First approach in the use of Rapid Assessment Methods (IDU-RAR)"
} 


\section{INTRODUCCIÓN}

La diseminación del VIH en el mapa mundial presenta variaciones importantes en cuanto a la vía de transmisión prevalente. Así, en África la transmisión sexual es la vía ordinaria de contagio, mientras en América las tasas son variables de país a país y aún de ciudad en ciudad. (MAP, 1997). Argentina, con $41 \%$ de las infecciones transmitidas por uso de drogas inyectables (UDI), es uno de los primeros países en América para esta vía (Ministerio de Salud, 1999). Los grandes centros urbanos de Argentina y Brasil concentran, en Sudamérica, la mayor parte de estos casos. En este sentido Rosario, con 1.300.000 habitantes, posee la mayor población VIH del país después de Capital y el conurbano bonaerense, representando poco más del $6 \%$ nacional. En la ciudad de Rosario la prevalencia de contagio VIH por UDI en los casos notificados desde 1988 hasta 1998 es de 72\% entre los varones. (Programa Municipal, 1997).

El contagio del VIH no constituye en absoluto la única causa de riesgo sanitario para los UDIs, existen otras infecciones y enfermedades no infecciosas, los daños sociales y legales, la disolución de los lazos familiares y parentales, entre otros. (Stimson, 1995).

EI UDI es una práctica ampliamente difundida en nuestra ciudad, sin embargo, la información local y nacional en materia de drogas es escasísima, y en materia de UDIs, prácticamente inexistente. En el presente estudio intentamos iniciar el armado epidemiológico de este campo, introduciendo las metodologías recomendadas en la Guía para la evaluación Rápida y desarrollo de Respuestas en el Uso de Sustancias Intravenosas (Rapid Assessment \& Response Method - IDU - RAR, 1998) del Programa sobre el Abuso de Sustancias de la OMS. (OMS, 1998).

\section{MATERIAL Y MÉTODOS:}

Se incluyó información de diversas publicaciones oficiales de VIH y se revisaron los estudios disponibles y publicaciones locales en busca de datos relacionados con el consumo de drogas inyectables. Se realizaron los siguientes estudios de campo: 40 encuestas directas, 15 entrevistas personales a UDIs y un rastreo ampliado por snow-ball con datos recogidos sobre más de 170 UDIs en un corte de cuatro meses, fueron sometidos a triangulación entre sí y relacionados con datos existentes.

Durante el segundo semestre de 1998 fueron realizadas 40 encuestas y 15 entrevistas en forma directa a pacientes consultantes en tres Servicios de consulta de adictos en las zonas norte, centro y sur de la ciudad. Dos Servicios eran públicos, uno privado; en el
90\% de los casos fue posible la confirmación serológica para VIH.

Entre los meses de septiembre y diciembre de 1998 realizamos un estudio utilizando la técnica de información nominativa (muestreo en red) a través de un snow-ball en el que participaron 10 pacientes regulares (7 masculinos y 3 femeninos) como informantes sanitarios directos, la cadena intermedia de información constó de alrededor de unas 15 personas más. La encuesta es simple de compilar y consta de sólo 7 preguntas. Obtuvimos datos depurados sobre 170 UDIs activos al momento del estudio. La composición geográfica final resultó heterogénea (36\% zona Norte y parte del cinturón Norte de la ciudad que recibe atención en Rosario, 13\% zona Centro, 31\% zona Sur y $20 \%$ zona Oeste) En los cálculos finales no intervienen los datos personales de los 10 encuestadores para no viciar la muestra (el grupo es íntegramente de consultantes regulares). A través del mismo método se recogieron datos sobre 80 usuarios crónicos de opiáceos. (Siri, Inchaurraga, 1999).

\section{RESULTADOS}

Para marzo de 1999 habían sido estimados oficialmente a nivel nacional 16.831 casos de HIV/SIDA. Estimaciones del Programa Nacional, OPS/OMS, y ONUSIDA calculaban en el informe de junio 1998 que entre 80.000 y 120.000 personas vivían con VIH en la Argentina. (ONUSIDA, 1998) Las cifras oficiales para Santa Fe son de 1021 casos, el 85\% de ellos aproximadamente reside en la ciudad de Rosario, representando la misma algo más del $6 \%$ de los casos. Si traspolamos las estimaciones reales a las cifras oficiales tenemos una proyección de $\mathbf{6 0 0 0}$ a 7000 casos de VIH/SIDA para Rosario. No obstante esta estimación, que consideramos conservadora, existen estudios que estiman en más de 10.000 el número de portadores en la ciudad. Dado que la tasa de prevalencia de transmisión sanguínea por UDI es de por lo menos de $60 \%$ (63\% es la tasa promedio combinada hombre/mujer en una relación 3:1,65\% es aquella que suma la sanguínea pura más la combinación con otras prácticas de riesgo, calculadas sobre datos del Inf. Epidemiológico de Promusida), el número de casos que habrían sido contagiados de VIH por el uso inyectable de drogas sería de almenos entre $\mathbf{3 6 0 0}$ a 4800 personas.

Según el resultado del estudio de muestreo en red sobre los 170 UDls aproximadamente el $65 \%$ de los mismos son $\mathrm{VIH}(+)$ (el $63 \%$ es con seguridad $\mathrm{VIH}(+)$, a los cuales debemos sumar una buena fracción del $12 \%$ que desconoce su estado serológico pero tiene diversas conductas de alto riesgo tales como UDI más prostitución o relaciones a riesgo con $\mathrm{VIH}(+)$, 
etc.). De tal modo el número total de UDIs con y sin VIH serían almenos $30 \%$ mayor que aquellos UDIs/VIH (+): el número total de UDIs en Rosario podría estimarse entonces entre 4000 a 6000.

Por supuesto es un número estimado, sujeto a la desviación posible en la transpolación y fundamentalmente al hecho de representar un número cumulativo, y por lo tanto estático, debido al desconocimiento de los índices dinámicos como la tasa de remisión espontánea o ligada a tratamiento así como la incidencia de la práctica UDI; sin embargo, nos parece una cifra bastante conforme a la realidad: entre 4000 y 6000 UDIs por lo menos habitan en la ciudad si consideramos aquellas personas que se han inyectado drogas intravenosas por lo menos una vez en los últimos cuatro meses. El número es mucho más alto si se toman en cuenta los antecedentes de uso intravenoso y no sólo el uso actual (UDIs acumulados). Nuestra población UDI es, además, difícil de estimar porque:

Nos hallamos en contacto con un porcentaje muy pequeño de UDIs. En nuestro estudio de muestreo en red el $\mathbf{8 9} \%$ de los UDI no concurría a ningún Servicio de Salud Mental, Psicología, Psiquiatría, Toxicología u otro para buscar tratamiento para su adicción: En cambio en Amsterdam por ejemplo, el Programa para Adicciones se halla en contacto con el $70 \%$ de los UDIs. (Buning, 1999)

- El patrón de utilización de nuestra principal sustancia de abuso, la cocaína, produce una tasa de recambio muy alta y veloz, continuamente están iniciándose en la práctica nuevos adictos a la vez que otros dejan, por diversos períodos de tiempo el uso intravenoso, a veces para siempre, creando una población de un número continuamente variable aunque en términos globales es estimable (en las entrevistas pueden escucharse claramente relatos acerca de este hecho, algunos pacientes pueden dejar de inyectarse por semanas, meses o a veces años y luego en determinado momento retomar el hábito). En Europa y Asia la adicción a opiáceos, forma inyectable predominante, tiende a producir una población de UDIs más estable, sostenida y prolongada en su hábito, debido al fuerte Síndrome de privación física que producen estas drogas.

Los datos surgidos a partir de la investigación en terreno son los siguientes.

Entre los 40 adictos encuestados (consultantes):

El $77 \%$ son varones y el $23 \%$ mujeres.

El $\mathbf{5 7} \%$ consulta por adicción a cocaína, el $\mathbf{3 7 \%}$ a las benzodiacepinas, el $14 \%$ a opiáceos y algo menos de un $10 \%$ a la marihuana, alrededor de $5 \%$ por inhalantes y otro $5 \%$ por adicción a anticolinérgicos.

El $63 \%$ registra antecedentes o uso actual de drogas i.v (37\% actual o menos de 6 meses, 16\% entre 6 meses y 2 años, y 10\% hace más de 2 años)

El $82 \%$ de los consultantes con antecedentes de UDI tiene antecedentes penales o causas penales abiertas

El $\mathbf{4 5 \%}$ de estos consultantes a Servicios por adicciones es VIH (+) en nuestra serie.

El $\mathbf{7 8 \%}$ había intentado tratamientos previos en diversos lugares, el $55 \%$ de los mismos es UDI (el $68,75 \%$ de los UDI que recibieron tratamientos anteriores tuvo entre 1 y 5 internaciones en Comunidades Terapéuticas).

Entre los 170 UDIs encuestados por muestreo de red:

El 70\% son varones y el 30\% mujeres.

El $63 \%$ es $\mathrm{VIH}(+)$, el $10 \%$ tiene altísimas probabilidades de serlo, aún cuando desconoce su estado serológico (comparte jeringas, combina homo/bisexualidad y/o prostitución sin protección), de modo que alrededor del $\mathbf{7 0} \%$ de esta serie de UDI es probablemente VIH (+) de ellos, el $\mathbf{6 2} \%$ no hace tratamiento para su VIH. Sólo un $20 \%$ hace tratamiento con control clínico regular. La extensa mayoría de los encuestados se encuentra fuera de tratamiento por adicción (el $\mathbf{8 9} \%$ no concurre a ningún Servicio de salud en busca de tratamiento para su adicción).

\begin{tabular}{|lr|}
\hline 20 años o menos: & $\mathbf{1 3 \%}$ \\
21 a 25 años: & $\mathbf{2 1 \%}$ \\
26 a 30 años: & $\mathbf{3 6 \%}$ \\
31 a 39 años: & $\mathbf{2 5 \%}$ \\
40 años o más: & $\mathbf{5 \%}$ \\
& Distribución etaria en el grupo $(n=150)$
\end{tabular}

Además, casi el $\mathbf{7 0} \%$ comparte y reutiliza equipo de inyección $(68 \% \pm 3 \%)$, sólo un $20 \%$ toma siempre precauciones.

El $100 \%$ de UDIs encuestados se inyecta cocaína y alrededor de un 14\% usa o ha usado opiáceos i.v (principalmente morfina) en forma generalmente irregular o esporádica con diferentes grados de frecuencia.

En un rastreo acotado con sólo dos informantes sanitarios se recogieron durante Enero de 1999 datos mínimos (con técnica nominativa) sobre un grupo de 80 adictos crónicos de Hydrocodona o dihidrocodeinona oral. De ellos, el $61 \%$ resultó VIH (+) y el $\mathbf{7 1 \%}$ consumía, además, cocaína intravenosa, el $89 \%$ se halla fuera de tratamiento para su adicción. Sin embargo, si tomamos el subgrupo de menores de 26 años (22 personas), encontramos que sólo el 23\% es UDI y el 23\% de los mismos es VIH (+).

Los opiáceos orales e inyectables son extensamente comercializados por una gran cantidad de 
bocas de venta, varias decenas de lugares, en la Zona Sur fundamentalmente, venden hidrocodona oral (a un precio corriente de 12 a 15 U\$S la dosis ( 5 cápsulas o comprimidos de $5 \mathrm{mg}$ ). La morfina en ampollas, aunque irregular en su aparición en el mercado, suele conseguirse con relativa frecuencia, sobre todo en ciertos círculos de Zonas Sur y Oeste de la ciudad, con precios de 5 a 10 U\$S la ampolla. Otros opiáceos pueden encontrarse con diversos grados de frecuencia.

La cocaína se encuentra prácticamente en todos los ámbitos metropolitanos, consiguiéndose con una facilidad geométrica (los puntos de ventas superan sin dudas el millar) y a precios bajísimos, tales como menos de 5 U\$S el gramo de clorhidrato de cocaína purísima. El uso inhalatorio se halla extendido a cantidades no estimadas pero seguramente altísimas, mucho mayores al uso intravenoso (5 ó 6 veces mayor como mínimo, lo cual hablaría de al menos un par de decenas de miles de usuarios regulares en la ciudad), este grupo tiene un alto porcentaje de peligro de entrar en abuso grave en cualquier momento, con graves repercusiones en su vida y su entorno.

Para ambos grupos es altamente frecuente la concurrencia de poliadicciones (combinaciones: cocaína + alcohol u opiáceos o benzodiacepinas o combinaciones de ellos, también se combinan las vías, por ejemplo opiáceos o benzodiacepinas orales + cocaína intravenosa, etc.) (Arbulu, 1996; Siri, 1999).

\section{DISCUSIÓN}

Creemos que el conocimiento preciso y la comprensión de las características antropológico-sociales, geográficas, etarias, de las patentes de consumo, sanitarias y subjetivas de los sujetos UDIs y otros usuarios de drogas es absolutamente imprescindible para poner en marcha las intervenciones más adecuadas y efectivas, así como los métodos idóneos para monitorearlas, tendientes a mejorar su estado en todos los sentidos.

Nuestro estudio comprende en suma, más de 170 UDIs que, para la cantidad estimada total, representan alrededor del $2 \%$ al $3 \%$. Por lo tanto creemos que la muestra adquiere significatividad estadística, de todos modos esperamos que estudios más extensos y perfeccionados confirmen o modifiquen y amplíen las conclusiones resultantes de éste, sobre todo en virtud de las diferencias existentes entre los diversos subgrupos que poseen características comunes propias. La utilización de la técnica de snow-ball por muestreo en red no arroja siempre datos con el nivel de precisión deseable, sin embargo, tiene una alta efectividad para acceder a grupos de muy difícil contacto. Por otro lado cabe la posibilidad de repetir el estudio con diver- sas poblaciones y hacer comparativos viendo si se llega al punto de saturación, lo que nos daría una idea clara de la certeza de los datos y la efectividad del método en particular. La ampliación de estudios directos sobre terreno así como el perfeccionamiento en las técnicas de evaluación de situación y respuesta a las intervenciones es una de las claves para el desarrollo de políticas adecuadas en materia de uso de drogas y sus riesgos. (Siri, Inchaurraga, 1999).

La concentración en torno a los 25 -30 años (36\%) conjugada con las entrevistas deja entrever una amplia franja de UDIs de vieja data (entre 5 y 10 años) que continúan después de tanto tiempo el uso intravenoso y constituyen un foco continuo de riesgo no sólo de VIH sino de otras infecciones debido a las pésimas condiciones en que se realizan las inyecciones y al bajísimo contacto con las estructuras sanitarias.

Es evidente en todos los estudios, el rol decisivo de los UDI en la epidemia de VIH/SIDA en la ciudad. La transmisión sanguínea no sólo afecta a los UDIs, sino que, además, incrementa las infecciones a través de sus parejas sexuales y sus hijos.

Pese a la inexistencia de pruebas epidemiológicas consistentes y definitivas algunos especialistas del tema refieren una disminución en las tasas de contagio por uso intravenoso de drogas. Dos estudios comparados, realizados en consultantes por adicciones a Servicios de Toxicología, mostraron una disminución en las consultas por UDI de 16\% en 1994 a sólo 3\% en 1997. (Sain, 1994, 1997) Tal disminución no se ve reflejada en la incidencia de los UDI en el VIH (siempre mayor al $30 \%$ ni se corresponde con la frecuencia con que los UDIs consultan en otros Servicios, por lo cual creemos necesario correlacionar este hecho con los tipos de abordajes o la oferta planteada (en el mismo estudio las consultas de pacientes con VIH disminuyó de un 32\% en 1994 a un 8\% en 1997). Aún si ello fuera cierto, aún si la incidencia directa actual de los UDIs en la transmisión no fuera del $60 \%$ sino que hubiese descendido a 35 o 30\% ello no nos eximiría en absoluto de implementar soluciones probadamente eficaces para lograr reducciones sustanciales y el mantenimiento de las mismas en la incidencia.

Este problema no fue ni es tomado adecuadamente en las políticas sanitarias, el UDI continúa siendo un fuerte tabú en el país de los UDIs.

\section{CONCLUSIONES}

Más allá de la epidemia de VIH, el UDI entraña una gran cantidad de riesgos, costos y daños para sí y la sociedad que ocurren diariamente ante nuestros ojos (clínicos o no) y para los cuales podrían tomarse medidas adecuadas. Existen medidas adecuadas y proba- 
das, como la reducción de daños. (O 'Hare, 1995) La reducción de daños es una política de salud que apunta a la disminución de las consecuencias negativas del consumo de drogas, a diferencia de la política llamada de abstinencia que comanda la política oficial Argentina en materia de drogas. Estas consecuencias, estos daños, pertenecen tanto al campo de lo social: marginación, criminalización, estigmatización que son los principales efectos de nuestra legislación que penaliza la tenencia de drogas para consumo personal, o sea, que penaliza el consumo. Y también pertenecen al campo de la salud: infecciones varias, hepatitis, sobredosis y fundamentalmente el SIDA. (Inchaurraga, 1995).

La reducción de daños abarca variados programas o estrategias que estan siendo aplicados en todo el mundo. Los más difundidos son los programas de cambio de jeringas y los programas de sustitución para los adictos a los opiáceos, ellos son también los programas más resistidos en Sudamérica, donde en la actualidad sólo Brasil los está implementando en algunos estados. (Castaño et al., 1998, Mesquita, 1999). Pero la reducción de daños abarca también la educación e información. Por una parte de los usuarios sobre las formas de desinfección de los equipos de inyección, riesgos de sobredosis y formas seguras de inyección. Por otro lado, de la comunidad general para evitar la marginación de estos sujetos y su consiguiente distanciamiento del sistema de salud. Incluye también el trabajo de calle para establecer contacto con la mayor cantidad posible de usuarios de drogas y la implementación de instituciones asitenciales públicas abiertas, con objetivos intermedios, dispuestas a escuchar y tratar a las personas esten o no abstinentes de drogas. (Inchaurraga, 1998).

Así, la Universidad Nacional de Rosario a partir de un convenio firmado con el Centro Regional de Salud Mental "Dr. Agudo Avila", ex Hospital Psiquiátrico de Rosario, creó el Servicio Asistencial para Drogadependientes. Este emprendimiento del Centro de Estudios Avanzados en Drogadependencias y SIDA (CEADS) de la UNR es a la fecha el único dispositivo asistencial en drogadependencias dependiente de la Universidad y uno de los dos efectores públicos especializados en la materia. Se trata de un Servicio inserto en el hospital público de salud mental de la ciudad, ya que se piensa a las drogadependencias como un problema de salud pública y, en tanto se considera que las mismas no constituyen un cuadro gnoseológico, un problema también de salud mental. Dicho servicio funciona con atención ambulatoria y en crisis, con orientación psicoanalítica y un equipo transdiciplinario.

Un enfoque de la salud pública que reivindica el rol del hospital y sitúa objetivos intermedios a la abstinencia, permite situar como prioridad la prevención del SIDA en estos pacientes. Una concepción de la prevención como participativa que incluye al sujeto como agente de salud con un rol activo, permite intervenir en la reducción de daños relacionados con el consumo de drogas, que a veces el sujeto no puede o no quiere interrumpir.

De las estrategias de reducción de daños en marcha en el Servicio, situaremos dos de los dispositivos que nos permiten dar cuenta de las posibilidades de trabajo en este sentido, que no sin dificultades han podido desarrollarse en el contexto de la política oficial en materia de drogas; represiva y abstencionista. Ellos son los grupos de reducción de daños y el programa piloto de sustitución. Situaremos también la necesidad de implementación de los programas de jeringas.

* Grupos de Reducción de daños: El trabajo clínico, caso por caso, sobre la posición subjetiva en relación a los riesgos y a la muerte se articula a una concepción de la salud pública que prioriza la reducción de daños ligados al consumo de drogas. Así se crearon espacios grupales que funcionan como talleres participativos de discusión y análisis de los daños relacionados con el consumo de drogas y los riesgos de transmisión del VIH/SIDA y sus posibilidades de prevención. En ellos, los sujetos en tanto que actores sociales, trabajan las representaciones sociales sobre el tema, aprender a desinfectar los equipos de inyección y elaboran mensajes sobre el no uso compartido de los equipos de inyección y la desinfección de agujas y jeringas.

En cuanto a la transmisión de información, sabemos que la misma no es suficiente y que cada uno hará lo que pueda con ella, lo que incluye en algunos casos, el no querer saber, pero también sabemos que la misma es necesaria. En relación a esto se sitúa una política de salud en el hospital público, que ocupa su lugar frente a la ausencia de mensajes para la prevención del VIH/SIDA en drogadependientes desde el discurso oficial.

En uno de los grupos, recientemente los sujetos construyeron el siguiente mensaje: "La jeringa es como el forro, se usa una sóla vez". Otro de los mensajes: "Cuando hay necesidad de droga, a veces nada importa, pero con el SIDA hay que pensar dos veces", se combinó en un folleto con la representación en el interior de cómo se desinfectan las agujas y las jeringas. Este mensaje se hace necesario en el contexto de la ausencia de programas de distribución de jeringas y ante la falta de accesibilidad a los equipos de inyección.

* Programa piloto de sustitución: Los programas de sustitución se hallan ampliamente difundidos y probados en el mundo para el tratamiento de la adicción crónica a opiáceos, y pueden proporcionar, además, un efecto adicional de disminución de consumo de cocaína en poliadictos (Fischer, 1998). En Rosario la elevada utilización de opiáceos es de vieja data, estudios en 1994 mostraban ya porcentajes cercanos al 
20\%. (Sain, 1994). Actualmente estamos llevando a cabo las primeras experiencias piloto en programas de sustitución. La sustitución es llevada a cabo con buprenorfina a bajas dosis $(0,4$ a $0,6 \mathrm{mg} /$ día sublinguales) y los resultados son hasta ahora altamente positivos, en concordancia con la experiencia mundial (disminución del consumo de opiáceos ilícitos y cocaína, disminución de la actividad delictiva y en la tasa de incidencia de seroconversión VIH, mejora en el status de salud, aumento de la resocialización, altas tasas de retención en tratamiento por largos plazos, disminución del sufrimiento subjetivo y las tensiones familiares, etc.) (Ministere Paris, 1997; Marks, 1997; Siri, 1999) El tratamiento se realiza en un marco de estricta prescripción controlada con apoyo de trabajo de farmacia, clínica, enfermería, psicología.

* Los programas de jeringas: Son hoy en Argentina un tema tabú, con debate cero a nivel social, apesar de que han probado, a través de más de quince años de experiencia mundial, europea, asiática y americana, que disminuye significativamente las tasas de transmisión VIH, sin que se registre un aumento del consumo de sustancias. (Rana, 1999; Wodak, 1998). Esta intervención no podrá, tarde o temprano, ser ignorada en una ciudad como la nuestra, con altísimos niveles de seropositividad VIH en UDIs y varios miles de adictos por vía intravenosa compartiendo equipo, inyectándose con agua de la canilla en el mejor de los casos, haciendo reutilización extensa de los mismos equipos de inyección que provocan abscesos e infecciones sangrantes y/o purulentas, endocarditis, flebitis, etc.

En 1996, Peter Lurie y Ernrest Drucker estimaron el número de infecciones por VIH asociadas con la oposición del gobierno de los Estados Unidos a los programas de jeringas. (Lurie y Drucker, 1997; Lurie 1998) Estimaron que entre 4.000 y 10.000 usuarios de drogas inyectables no se habrían infectado con el VIH en los Estados Unidos, si hubieran tenido acceso a "jeringas limpias." Podríamos preguntarnos con temor qué resultados podrían darnos estudios similares en nuestro país, o en nuestra ciudad; una ciudad con una vieja y profunda tradición en relación al uso intravenoso de drogas, conocida en el Cono Sur por poseer una de las tasas de seropositividad para VIH por vía de transmisión sanguínea más alta de Sudamérica.

Es de señalar la importancia de estos programas en las poblaciones usuarias de cocaína, ya que estas personas llegan a inyectarse hasta veinte veces al día, multiplicándose así los riesgos y los daños. Así hemos iniciado en 1998 un debate científico y público con información correcta y objetiva, que se ha profundizado recientemente con una campaña de información y sensibilización sobre las problemáticas de los usuarios de drogas inyectables y los programas de jeringas y un petitorio de apoyo a los mismos, para llegar lo más pronto posible a un consenso sobre la forma de imple- mentarlos con mayor efectividad y menores obstáculos (como el avance en el campo legal, y la aceptabilidad social) en Argentina.

Las medidas de reducción de daños aportan muchas intervenciones útiles en el campo de las adicciones, en sociedades como la nuestra donde los daños relacionados con las condiciones en que los sujetos consumen drogas se han demostrado mayores que los ocasionados por la droga misma. (Inchaurraga, 1999). La base de ellas la constituye el consenso social y político, que permitan un encuadre legislativo y policial tal que soporte y apoye, en vez de perjudicar y obstaculizar, la situación social e individual de los consumidores de drogas. Justamente la sanción sobre la libertad de los consumidores es ya uno de los principales daños que se pueden ocasionar en el actual marco legislativo. La ley debería también ser clara en lo relativo a permitir y fomentar la puesta en marcha de programas como los de intercambio de jeringas, tan necesarios en ciudades con muchos UDIs con elevadísimas tasas de seropositividad VIH como la nuestra, y en acabar finalmente con la cuestión de que hablar claramente de las drogas sea hacer su apología. El modelo de reducción de daños no hace apología del uso de drogas.

Indudablemente la reciente constitución de la Asociación de Reducción de Daños de la Argentina (ARDA) con participación de representantes de diversas ciudades y regiones del país nos ayudará a desarrollar iniciativas en reducción de daños y a fortalecer y multiplicar experiencias solitarias, como la de Rosario.

\section{REFERENCIAS BIBLIOGRÁFICAS}

M., Biglione J. (1996) Los jovenes Rosarinos, un nuevo grupo de riesgo. Sidanews. Internet.

Benetucci, J. (1996) “Aspectos clínico-epidemiológicos de la infección por HIV/SIDA" en "Drogadependencias; reflexiones sobre el sujeto y la cultura" pag. 113 - 121. Silvia Inchaurraga Comp. Ed. Homo Sapiens. Rosario.

Boletín sobre el SIDA en la Argentina. Ministerio de Salud y Acción Social. Programa Nacional de lucha contra los retrovirus del Humano, SIDA y ETS (1998) No 14, Julio y Marzo de 1999.

Buning E. (1999) “Un abordaje de salud pública al problema de la droga. Actividades del Servicio de Salud municipal de Amsterdam" en "Drogas y políticas públicas. El modelo de reducción de daños" S. Inchaurraga Comp. Ed. CEADS-UNR.

Castaño Pérez G., Fresco M , Lo Valvo R.. (1998) "Workshop: Reducción de Daños en Sudamérica" (1998) $9^{\circ}$ International Conference on the Reduction of Drug Related Harm, San Pablo, Brasil, 15 -19 Mar.1998. 
Fischer B., (1998) "Profile of untreated and illicit opiate users in Toronto, Canadá". 9० Conf. on the Reduction of Drug Related Harm, Sao Paulo, Brasil. 15 al 19 de Marzo de 1998.

Inchaurraga S. "EI SIDA en la Cultura” . (1995) Ed. Homo Sapiens, Rosario, Argentina.

Inchaurraga S. (1999) “Drogas y políticas públicas. El modelo de reducción de daños". Ed. CEADS-UNR, Rosario.

Inchaurraga S. Vírgala E. (1998) "How to reduce harm within the argentine drug policy" en "Epidemiology and Prevention". Ed. Monduzzi, Bologna,

Informe Epidemiológico sobre VIH/SIDA (1997) Programa Municipal de SIDA. Departamento de Epidemiología. Secretaría de Salud Municipalidad de Rosario.

Lurie P., (1998) “Quando a ciência e a política colidem: A resposta do governo federal dos EUA aos Programas de troca de seringas" en "Troca de seringas, droga e AIDS", Ministero da Saúde. Brasilia.

Lurie P., Drucker E (1997) "An opportunity lost: HIV infections associated with the lack of a national needle exchange programme in the United States."The Lancet, 394, pág. 604-8.

Marks J., Dosagem de manutençao de heroína e cocaína. En “Drogas Hegemonia do Cinismo". Brasilia, 1997.

MAP - Monitoring the AIDS Pandemic. Informe Final. Estado y Tendencia de las Epidemias del VIH/SIDA en América Latina y el Caribe. Lima, 3 - 6 Dic. 1997.

Ministère de la Santé. (1997) "Médecine et toxicomanes. Traitements de substitution des pharmaco-dependances aux opiacés". Paris.

Mesquita F. (1999) "Dar oportunidad de vivir al usuario de drogas inyectables" en "Drogas y Políticas Públicas. El
Modelo de Reducción de daños" S. Inchaurraga Comp. CEADS Edit., Rosario.

O’Hare P. (1995), "Apuntes sobre el concepto de reducción de daños" en "La reducción de los daños relacionados con las drogas" Grup Igia. Barcelona.

OMS, (1998) Guía para la valoración rápida sobre el uso de drogas endovenosas.

ONUSIDA. La epidemia de SIDA: situación en Diciembre de 1998.

Rana S. (1999) "Experiencias de un programa de cambio de jeringas para usuarios inyectables en Asia" en "Drogas y Políticas Públicas. El Modelo de Reducción de daños " de Inchaurraga S. Comp. Edit. CEADS, Rosario.

Saín O. et al. (1994 y 1997), "Consultas por farmacodependencia a Toxicología". Área VIII Salud, Rosario-Sta.FeArgentina.

Siri P. , Inchaurraga S. (1999) “Epidemiologic profile of drug addiction on IDU population in Rosario city, Argentina: First approach in the use of Rapid Assessmnt Methods (RAR)" 10 International Conference on the Reduction of Drug Related Harm, Ginebra .

Siri P. (1999) "Estimación cuali-cuantitativa de las adicciones intravenosas en Rosario" en "Drogas y políticas públicas. El modelo de Reducción de Dañoss" Silvia Inchaurraga Comp. Ed. CEADS-UNR

Stimson G. (1995) "AIDs and IVDU in UK, 1987-1993. Prevention of epidemy and Public Politics." Social and Medicine 41.

Wodak, A. (1998) "Reduçao de Danos e Programas de Troca de Seringas" En Troca de Seringas, Drogas e AIDS. Brasilia. 
